\title{
An error estimator for transmitting boundary conditions in fluid-structure interaction problems
}

\author{
N. Bouaanani \& B. Miquel \\ Department of Civil, Geological and Mining Engineering, \\ Ecole Polytechnique de Montreal, Canada
}

\begin{abstract}
This paper proposes error estimators to validate Transmitting Boundary Conditions (TBCs) in fluid-structure interaction problems. The error estimators are based on a new formulation of the dynamic response of fluid-structure systems including TBCs. The mathematical background is briefly discussed and the obtained equations are solved numerically to assess the accuracy of a given TBC and determine the associated error independently of FEM or BEM modeling of the fluid domain. The error estimators take account of : (i) structure's flexibility, (ii) fluid compressibility, (iii) energy dissipation at fluid boundaries, (iv) fluid domain truncation length, and (v) excitation frequency. An illustrative example consisting of a dam-reservoir system is presented where an error estimator is used to evaluate the effects of various TBCs on the hydrodynamic pressure acting on the dam upstream face. The proposed formulation can be programmed easily and used efficiently for rigorous assessment of classical or newly-developed TBCs for vibrating fluid-structure systems.

Keywords: fluid-structure interaction, transmitting boundary conditions, error analysis, analytical formulations, dam-reservoir systems, earthquake analysis, hydrodynamic loading.
\end{abstract}

\section{Introduction}

Accurate evaluation of hydrodynamic loading on structures such as dams is crucial for their safety assessment. Significant research has been devoted to study this type of loading since the pioneering work of Westergaard [1] who introduced the 
added mass concept. Since then, more advanced techniques have been developed for complex fluid-structure interaction problems with the Finite Element Method (FEM) being the most popular [2-4]. Practical FEM modeling requires the virtual truncation of large extent or infinite fluid domains at a finite distance from the studied structure. In this case, a Transmitting Boundary Condition (TBC) has to be applied at the boundary between the near and far fields to ensure adequate energy radiation $[5,6]$. These special boundary conditions and the truncation distance should be defined appropriately to prevent reflection of spurious waves back towards the structure. Otherwise, significant errors may be introduced in the prediction of the dynamic response of the fluid-structure system.

TBCs are generally approximate, thus introducing some error into the solution. This error is usually minimized based on the experience and judgement of the analyst after some initial guess of the truncation boundary location. Successive trials are then performed to ensure numerical convergence of the FEM solutions as a function of truncation length and mesh refinement. It is important however to separate errors that can be attributed strictly to the type of TBC applied and its location from the discretization, convergence, or other numerical or modeling errors specific to the FEM packages used. The main objective of this work is to propose an original analytical technique to reliably predict the accuracy of a given TBC in a vibrating fluid-structure system and estimate the TBC effects and associated error independently of FEM modeling of the fluid domain.

\section{New formulation for TBC error estimators}

Fig. 1 illustrates a schematic structure of height $H_{\mathrm{s}}$, in contact with a semi-infinite fluid reservoir of constant height $H_{\mathrm{r}}$, truncated at a distance $L_{\mathrm{r}}$ from the fluidstructure interface. A Cartesian coordinate system with axes $x$ and $y$ and origin at the heel of the structure is adopted as well as the following main assumptions : (i) the structure and the fluid are assumed to have a linear elastic behavior; (ii) the fluid is compressible and inviscid, with its motion irrotational and limited to small amplitudes; and (iii) gravity surface waves are neglected. Under these assumptions, the hydrodynamic pressure $p(x, y, t)$ within the fluid domain obeys the wave equation

$$
\nabla^{2} p=\frac{1}{C_{\mathrm{r}}^{2}} \frac{\partial^{2} p}{\partial t^{2}}
$$

where $\nabla^{2}$ is the Laplace differential operator, $t$ the time variable, $\rho_{\mathrm{r}}$ the mass density of water and $C_{\mathrm{r}}$ the velocity of compression waves in the fluid domain. Considering horizontal harmonic ground accelerations $\ddot{u}_{\mathrm{g}}(t)=a_{\mathrm{g}} \mathrm{e}^{\mathrm{i} \omega t}$, hydrodynamic pressure can be expressed in the frequency domain as $p(x, y, t)=$ $\bar{p}(x, y, \omega) \mathrm{e}^{\mathrm{i} \omega t}$, where $\bar{p}(x, y, \omega)$ is a complex-valued frequency response function (FRF). Introducing this transformation into eqn (1) yields the classical Helmholtz equation

$$
\nabla^{2} \bar{p}+\frac{\omega^{2}}{C_{\mathrm{r}}^{2}} \bar{p}=0
$$




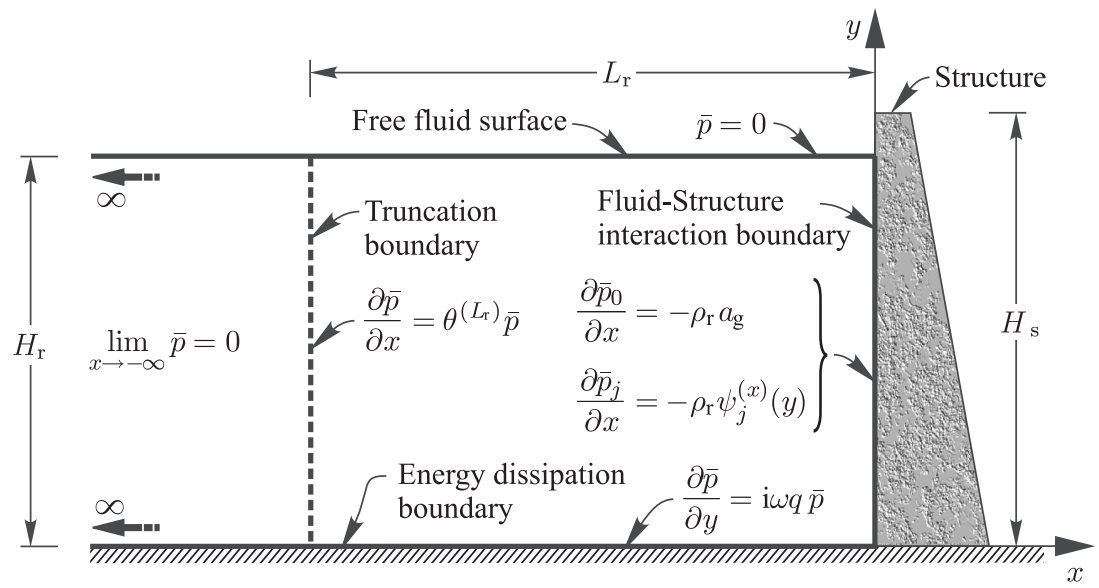

Figure 1: Fluid-structure system and boundary conditions.

We show that the hydrodynamic pressure FRF $\bar{p}$ can be decomposed as [7]

$$
\bar{p}(x, y, \omega)=\bar{p}_{0}(x, y, \omega)-\omega^{2} \sum_{j=1}^{m_{\mathrm{s}}} \bar{Z}_{j}(\omega) \bar{p}_{j}(x, y, \omega)
$$

where $\bar{p}_{0}$ is the FRF for hydrodynamic pressure due to rigid body motion of the structure, and where $\bar{p}_{j}$ is the FRF corresponding to hydrodynamic pressure due to horizontal acceleration $\psi_{j}^{(x)}(y)=\psi_{j}^{(x)}(0, y)$ of the fluid-structure interface along structural mode $j$. The boundary conditions (BCs) to be satisfied by FRFs $\bar{p}_{0}$ and $\bar{p}_{j}$ for the semi-infinite and truncated fluid-domain cases are illustrated in fig. 1, including a free surface $\mathrm{BC}$, a fluid-structure interaction $\mathrm{BC}$, an infinite $\mathrm{BC}$, a TBC and a dissipative $\mathrm{BC}$ defined using a damping coefficient $q$. In the rest of the paper, a superscript $(\infty)$ will be used to denote classical hydrodynamic pressures corresponding to a semi-infinite fluid-domain [7]. These pressures are written here as the summation of $m_{\mathrm{r}}$ functions $\bar{p}_{0 n}^{(\infty)}$ and $\bar{p}_{j n}^{(\infty)}$ corresponding each to a reservoir mode $n$

$$
\bar{p}_{0}^{(\infty)}(x, y, \omega)=\sum_{n=1}^{m_{\mathrm{r}}} \bar{p}_{0 n}^{(\infty)}(x, y, \omega) ; \quad \bar{p}_{j}^{(\infty)}(x, y, \omega)=\sum_{n=1}^{m_{\mathrm{r}}} \bar{p}_{j n}^{(\infty)}(x, y, \omega)
$$

in which

$$
\begin{gathered}
\bar{p}_{0 n}^{(\infty)}(x, y, \omega)=-2 \rho_{\mathrm{r}} a_{\mathrm{g}} H_{\mathrm{r}} \frac{\lambda_{n}^{2}(\omega)}{\beta_{n}(\omega)} \frac{I_{0 n}(\omega)}{\kappa_{n}(\omega)} \mathrm{e}^{\kappa_{n}(\omega) x} Y_{n}(y, \omega) \\
\bar{p}_{j n}^{(\infty)}(x, y, \omega)=-2 \rho_{\mathrm{r}} H_{\mathrm{r}} \frac{\lambda_{n}^{2}(\omega)}{\beta_{n}(\omega)} \frac{I_{j n}(\omega)}{\kappa_{n}(\omega)} \mathrm{e}^{\kappa_{n}(\omega) x} Y_{n}(y, \omega)
\end{gathered}
$$

where $\lambda_{n}$ and $Y_{n}$ are complex-valued frequency dependent eigenvalues and orthogonal eigenfunctions satisfying for each fluid vibration mode $n$ 


$$
\begin{gathered}
\mathrm{e}^{2 \mathrm{i} \lambda_{n}(\omega) H_{\mathrm{r}}}=-\frac{\lambda_{n}(\omega)-\omega q}{\lambda_{n}(\omega)+\omega q} \\
Y_{n}(y, \omega)=\frac{\left[\lambda_{n}(\omega)-\omega q\right] \mathrm{e}^{-\mathrm{i} \lambda_{n}(\omega) y}+\left[\lambda_{n}(\omega)+\omega q\right] \mathrm{e}^{\mathrm{i} \lambda_{n}(\omega) y}}{2 \lambda_{n}(\omega)}
\end{gathered}
$$

and where the terms $\beta_{n}, \kappa_{n}, I_{0 n}, I_{j n}$ are given by

$$
\begin{array}{r}
\beta_{n}(\omega)=H_{\mathrm{r}}\left[\lambda_{n}^{2}(\omega)-\omega^{2} q^{2}\right]+\mathrm{i} \omega q ; \quad \kappa_{n}(\omega)=\sqrt{\lambda_{n}^{2}(\omega)-\frac{\omega^{2}}{C_{\mathrm{r}}^{2}}} \\
I_{0 n}(\omega)=\frac{1}{H_{\mathrm{r}}} \int_{0}^{H_{\mathrm{r}}} Y_{n}(y, \omega) \mathrm{d} y ; \quad I_{j n}(\omega)=\frac{1}{H_{\mathrm{r}}} \int_{0}^{H_{\mathrm{r}}} \psi_{j}^{(x)}(y) Y_{n}(y, \omega) \mathrm{d} y
\end{array}
$$

As mentioned previously, appropriate TBCs are required for efficient finite element modeling of fluid-structure systems. In this case, infinie boundary conditions are replaced by TBCs to be applied at a finite distance $L_{\mathrm{r}}$ from the fluidstructure interface as shown in fig. 1. TBCs are used to prevent or reduce reflection of waves impinging a fictitious truncation boundary of an infinite fluid domain. They can generally be defined by the relationship between the hydrodynamic pressure and its normal gradient both determined at the truncation boundary. According to the system of axes in fig. 1, we consider TBCs that can be expressed as

$$
\begin{aligned}
& \frac{\partial \bar{p}_{0}^{(\infty)}}{\partial x}\left(-L_{\mathrm{r}}, y, \omega\right)=\theta_{0}^{\left(L_{\mathrm{r}}\right)}(y, \omega) \bar{p}_{0}^{(\infty)}\left(-L_{\mathrm{r}}, y, \omega\right) \\
& \frac{\partial \bar{p}_{j}^{(\infty)}}{\partial x}\left(-L_{\mathrm{r}}, y, \omega\right)=\theta_{j}^{\left(L_{\mathrm{r}}\right)}(y, \omega) \bar{p}_{j}^{(\infty)}\left(-L_{\mathrm{r}}, y, \omega\right)
\end{aligned}
$$

where the functions $\theta_{0}^{\left(L_{\mathrm{r}}\right)}$ and $\theta_{j}^{\left(L_{\mathrm{r}}\right)}$ are generally height- and frequencydependent. We note that the TBC in eqn (11) accounts for rigid body motion of the dam, while that in eqn (12) accounts for dam elastic deformation along structural mode shape $\psi_{j}^{(x)}$. In the rest of the paper, a superscript ${ }^{\left(L_{\mathrm{r}}\right)}$ will be used to denote hydrodynamic pressures obtained using TBCs placed at a distance $L_{\mathrm{r}}$ from fluidstructure interface. In this case, eqns (4) to (6) of the classical formulation are no longer valid. To get a rigorous assessment of the accuracy and effectiveness of a given TBC, a new formulation is developed in this work. The hydrodynamic pressures $\bar{p}_{0}^{\left(L_{\mathrm{r}}\right)}$ and $\bar{p}_{j}^{\left(L_{\mathrm{r}}\right)}$ are first written as the summation of $m_{\mathrm{r}}$ functions $\bar{p}_{0 n}^{\left(L_{\mathrm{r}}\right)}$ and $\bar{p}_{j n}^{\left(L_{\mathrm{r}}\right)}$ corresponding each to a reservoir mode $n$

$$
\bar{p}_{0}^{\left(L_{\mathrm{r}}\right)}(x, y, \omega)=\sum_{n=1}^{m_{\mathrm{r}}} \bar{p}_{0 n}^{\left(L_{\mathrm{r}}\right)}(x, y, \omega) ; \quad \bar{p}_{j}^{\left(L_{\mathrm{r}}\right)}(x, y, \omega)=\sum_{n=1}^{m_{\mathrm{r}}} \bar{p}_{j n}^{\left(L_{\mathrm{r}}\right)}(x, y, \omega)
$$

In this work, we show that the FRFs $\bar{p}_{0 n}^{\left(L_{\mathrm{r}}\right)}$ and $\bar{p}_{j n}^{\left(L_{\mathrm{r}}\right)}$ can be expressed as 


$$
\begin{aligned}
& \bar{p}_{0 n}^{\left(L_{\mathrm{r}}\right)}(x, y, \omega)=\left[\mathrm{e}^{-\kappa_{n}(\omega) x}+\mathrm{e}^{\kappa_{n}(\omega) x}\right] \Gamma_{n}^{(0)}(\omega) Y_{n}(y, \omega)+\bar{p}_{0 n}^{(\infty)}(x, y, \omega) \\
& \bar{p}_{j n}^{\left(L_{\mathrm{r}}\right)}(x, y, \omega)=\left[\mathrm{e}^{-\kappa_{n}(\omega) x}+\mathrm{e}^{\kappa_{n}(\omega) x}\right] \Gamma_{n}^{(j)}(\omega) Y_{n}(y, \omega)+\bar{p}_{j n}^{(\infty)}(x, y, \omega)
\end{aligned}
$$

in which $\Gamma_{n}^{(0)}(\omega)$ and $\Gamma_{n}^{(j)}(\omega)$ are elements of vectors $\Gamma^{(0)}$ and $\Gamma^{(j)}$, obtained by solving the systems of linear equations

$$
\mathbf{A}^{(0)}(\omega) \boldsymbol{\Gamma}^{(0)}(\omega)=\mathbf{B}^{(0)}(\omega) ; \quad \mathbf{A}^{(j)}(\omega) \boldsymbol{\Gamma}^{(j)}(\omega)=\mathbf{B}^{(j)}(\omega)
$$

where the elements of matrix $\mathbf{A}^{(\ell)}$ and vector $\mathbf{B}^{(\ell)}$ are given for $\ell=0, j$ by

$$
\begin{aligned}
& A_{s n}^{(\ell)}(\omega)= \frac{\kappa_{n}(\omega) \beta_{n}(\omega)}{2 \lambda_{n}^{2}(\omega)}\left[\mathrm{e}^{-\kappa_{n}(\omega) L_{\mathrm{r}}}-\mathrm{e}^{\kappa_{n}(\omega) L_{\mathrm{r}}}\right] \delta_{s n} \\
&-\left[\mathrm{e}^{-\kappa_{n}(\omega) L_{\mathrm{r}}}+\mathrm{e}^{\kappa_{n}(\omega) L_{\mathrm{r}}}\right] \int_{0}^{H_{\mathrm{r}}} \theta_{\ell}^{\left(L_{\mathrm{r}}\right)}(y, \omega) Y_{s}(y, \omega) Y_{n}(y, \omega) \mathrm{d} y \\
& B_{s}^{(\ell)}(\omega)=2 \rho_{\mathrm{r}} \eta_{\ell} H_{\mathrm{r}} \sum_{n=1}^{m_{\mathrm{r}}} \frac{\lambda_{n}^{2}(\omega)}{\beta_{n}(\omega)} \frac{I_{\ell n}(\omega)}{\kappa_{n}(\omega)}\left[\frac{\kappa_{n}(\omega) \beta_{n}(\omega)}{2 \lambda_{n}^{2}(\omega)} \delta_{s n}\right. \\
&\left.-\int_{0}^{H_{\mathrm{r}}} \theta_{\ell}^{\left(L_{\mathrm{r}}\right)}(y, \omega) Y_{s}(y, \omega) Y_{n}(y, \omega) \mathrm{d} y\right] \mathrm{e}^{-\kappa_{n}(\omega) L_{\mathrm{r}}}
\end{aligned}
$$

in which $\eta_{0}=a_{\mathrm{g}}, \eta_{1}=1$ and $\delta$ is the Kronecker symbol.

One important aspect of this formulation is that it takes account of the variations of the functions $\theta_{0}^{\left(L_{\mathrm{r}}\right)}$ and $\theta_{j}^{\left(L_{\mathrm{r}}\right)}$ over the height of the fluid domain. We also prove that if these functions were assumed constant over the height of the fluid domain, i.e. independent of the $y$ coordinate, eqns (14) and (15) simplify to

$$
\begin{gathered}
\bar{p}_{0 n}^{\left(L_{\mathrm{r}}\right)}(x, y, \omega)=-2 \rho_{\mathrm{r}} a_{\mathrm{g}} H_{\mathrm{r}} \frac{\lambda_{n}^{2}(\omega)}{\beta_{n}(\omega)} \frac{I_{0 n}(\omega)}{\kappa_{n}(\omega)} X_{0 n}^{\left(L_{\mathrm{r}}\right)}(x, \omega) Y_{n}(y, \omega) \\
\bar{p}_{j n}^{\left(L_{\mathrm{r}}\right)}(x, y, \omega)=-2 \rho_{\mathrm{r}} H_{\mathrm{r}} \frac{\lambda_{n}^{2}(\omega)}{\beta_{n}(\omega)} \frac{I_{j n}(\omega)}{\kappa_{n}(\omega)} X_{j n}^{\left(L_{\mathrm{r}}\right)}(x, \omega) Y_{n}(y, \omega)
\end{gathered}
$$

where $X_{\ell n}^{\left(L_{\mathrm{r}}\right)}$ and $\zeta_{\ell n}^{\left(L_{\mathrm{r}}\right)}$ are given for $\ell=0, j$ by

$$
\begin{gathered}
X_{\ell n}^{\left(L_{\mathrm{r}}\right)}(x, \omega)=\frac{\mathrm{e}^{\kappa_{n}(\omega)\left(x+L_{\mathrm{r}}\right)}+\zeta_{\ell n}^{\left(L_{\mathrm{r}}\right)}(\omega) \mathrm{e}^{-\kappa_{n}(\omega)\left(x+L_{\mathrm{r}}\right)}}{\mathrm{e}^{\kappa_{n}(\omega) L_{\mathrm{r}}}-\zeta_{\ell n}^{\left(L_{\mathrm{r}}\right)}(\omega) \mathrm{e}^{-\kappa_{n}(\omega) L_{\mathrm{r}}}} \\
\zeta_{\ell n}^{\left(L_{\mathrm{r}}\right)}(\omega)=\frac{\kappa_{n}(\omega)-\theta_{\ell}^{\left(L_{\mathrm{r}}\right)}(\omega)}{\kappa_{n}(\omega)+\theta_{\ell}^{\left(L_{\mathrm{r}}\right)}(\omega)}
\end{gathered}
$$


Depending on whether an infinite condition or a TBC is applied, the FRF for total hydrodynamic pressure is then given by

$$
\begin{aligned}
& \bar{p}^{(\infty)}(x, y, \omega)=\bar{p}_{0}^{(\infty)}(x, y, \omega)-\omega^{2} \sum_{j=1}^{m_{\mathrm{s}}} \bar{Z}_{j}^{(\infty)}(\omega) \bar{p}_{j}^{(\infty)}(x, y, \omega) \\
& \bar{p}^{\left(L_{\mathrm{r}}\right)}(x, y, \omega)=\bar{p}_{0}^{\left(L_{\mathrm{r}}\right)}(x, y, \omega)-\omega^{2} \sum_{j=1}^{m_{\mathrm{s}}} \bar{Z}_{j}^{\left(L_{\mathrm{r}}\right)}(\omega) \bar{p}_{j}^{\left(L_{\mathrm{r}}\right)}(x, y, \omega)
\end{aligned}
$$

where the vectors $\overline{\mathbf{Z}}^{(\infty)}$ and $\overline{\mathbf{Z}}^{\left(L_{\mathrm{r}}\right)}$ of generalized coordinates $\bar{Z}_{j}^{(\infty)}$ and $\bar{Z}_{j}^{\left(L_{\mathrm{r}}\right)}, j=$ $1 \ldots m_{\mathrm{s}}$, are solutions of the system of equations

$$
\overline{\mathbf{S}}^{(\infty)} \overline{\mathbf{Z}}^{(\infty)}=\overline{\mathbf{Q}}^{(\infty)} ; \quad \quad \overline{\mathbf{S}}^{\left(L_{\mathrm{r}}\right)} \overline{\mathbf{Z}}^{\left(L_{\mathrm{r}}\right)}=\overline{\mathbf{Q}}^{\left(L_{\mathrm{r}}\right)}
$$

in which elements of $\overline{\mathbf{S}}^{(\infty)}, \overline{\mathbf{S}}^{\left(L_{\mathrm{r}}\right)}, \overline{\mathbf{Q}}^{(\infty)}$ and $\overline{\mathbf{Q}}^{\left(L_{\mathrm{r}}\right)}$ are obtained for $n=1 \ldots m_{\mathrm{S}}$ and $j=1 \ldots m_{\mathrm{s}}$ as

$$
\begin{gathered}
\bar{S}_{n j}^{(\infty)}(\omega)=\left[-\omega^{2}+\left(1+\mathrm{i} \eta_{\mathrm{s}}\right) \omega_{n}^{2}\right] \delta_{n j}+\omega^{2} \int_{0}^{H_{\mathrm{r}}} \bar{p}_{j}^{(\infty)}(0, y, \omega) \psi_{n}^{(x)}(y) \mathrm{d} y \\
\bar{Q}_{n}^{(\infty)}(\omega)=-a_{\mathrm{g}} \boldsymbol{\psi}_{n}^{\mathrm{T}} \mathbf{M} \mathbf{1}+\int_{0}^{H_{\mathrm{r}}} \bar{p}_{0}^{(\infty)}(0, y, \omega) \psi_{n}^{(x)}(y) \mathrm{d} y
\end{gathered}
$$

where $\omega_{n}$ is the vibration frequency corresponding to structural mode shape $\psi_{n}$ and $\eta_{\mathrm{s}}$ is a assumed constant hysteretic damping of the structure. A convergence study is conducted to determine the sufficient numbers $m_{\mathrm{s}}$ and $m_{\mathrm{r}}$ of structural and reservoir mode shapes to be included into each specific analysis. Using the equations presented above, error estimators to monitor the sensitivity of various response quantities to a given TBC can be defined. Due to space limitations, only the hydrodynamic pressure error estimator defined by

$$
\eta_{|\bar{p}|}^{\left(L_{\mathrm{r}}\right)}(y, \omega)=\frac{\left|\bar{p}^{\left(L_{\mathrm{r}}\right)}(0, y, \omega)\right|-\left|\bar{p}^{(\infty)}(0, y, \omega)\right|}{\left|\bar{p}^{(\infty)}(0, y, \omega)\right|}
$$

is investigated in the next section.

\section{Application to a dam-reservoir system}

For illustration purposes, the formulation and error estimator presented previously are used to study the dam-reservoir system shown in fig. 2, subjected to a unit horizontal harmonic ground acceleration $\ddot{u}_{\mathrm{g}}(t)=\mathrm{e}^{\mathrm{i} \omega t}$. The simplified dam crosssection has a height $H_{\mathrm{s}}=121.92 \mathrm{~m}(400 \mathrm{ft})$, a downstream slope of 0.8 and a vertical upstream face. A full reservoir is assumed, i.e. $H_{\mathrm{r}}=H_{\mathrm{s}}$, and different reservoir truncation lengths $L_{\mathrm{r}}$ are considered as will be discussed later. Fig. 2 illustrates the boundary conditions used. A dam Poisson's ratio $\nu_{\mathrm{s}}=0.2$ and mass density $\rho_{\mathrm{s}}=2400 \mathrm{~kg} / \mathrm{m}^{3}$ are adopted. To assess the influence of dam stiffness, 


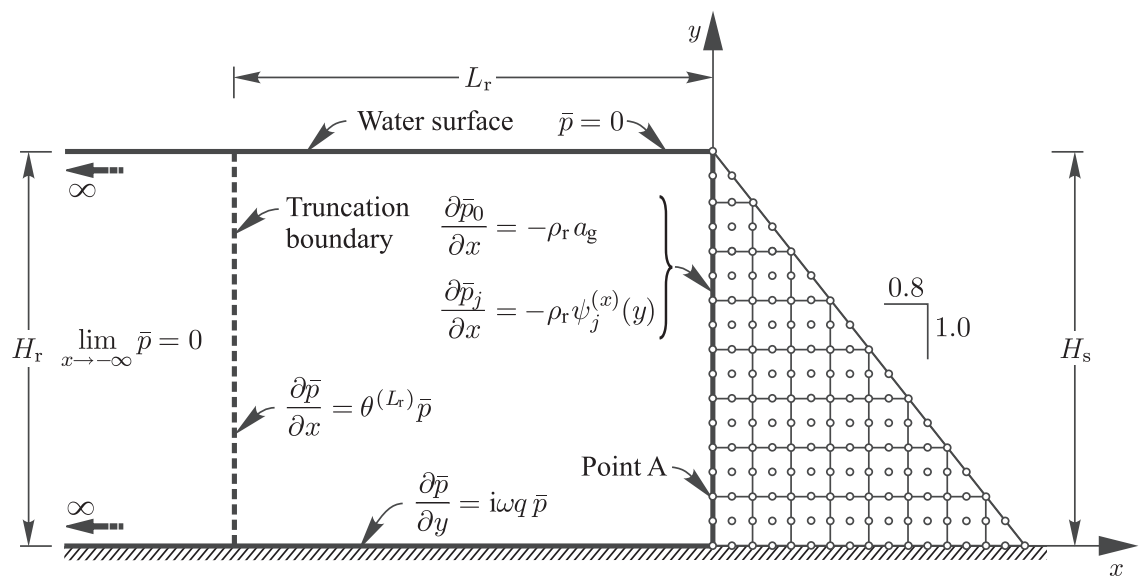

Figure 2: Dam-reservoir system studied and boundary conditions used.

two moduli of elasticity $E_{\mathrm{s}}=25 \mathrm{GPa}$ and $E_{\mathrm{s}}=35 \mathrm{GPa}$ are considered. A constant structural hysteretic damping factor $\eta_{\mathrm{s}}=0.1$ is adopted. The water is assumed compressible, with a velocity of pressure waves $C_{\mathrm{r}}=1440 \mathrm{~m} / \mathrm{s}$ and a mass density $\rho_{\mathrm{r}}=1000 \mathrm{~kg} / \mathrm{m}^{3}$. To obtain the mode shapes $\psi_{j}, j=1 \ldots m_{\mathrm{s}}$, the dam section is modeled using quadrilateral 9-node and triangular 6-node plane stress finite elements as illustrated in fig. 2.

The hydrodynamic pressure error estimator $\eta_{|\bar{p}|}^{\left(L_{\mathrm{r}}\right)}$ is determined at height $y_{\mathrm{A}}=$ $0.1 H_{\mathrm{r}}$ for the following TBCs:

- Sommerfeld radiation boundary condition [8], corresponding to

$$
\theta_{0}^{\left(L_{\mathrm{r}}\right)}(\omega)=\theta_{j}^{\left(L_{\mathrm{r}}\right)}(\omega)=\frac{\mathrm{i} \omega}{C_{\mathrm{r}}}
$$

- Sharan boundary condition [3], corresponding to

$$
\theta_{0}^{\left(L_{\mathrm{r}}\right)}(\omega)=\theta_{j}^{\left(L_{\mathrm{r}}\right)}(\omega)=\kappa_{1}(\omega)
$$

- A proposed analytical TBC given by

$$
\tilde{\theta}_{\ell}^{\left(L_{\mathrm{r}}\right)}(y, \omega)=\frac{\sum_{n=1}^{\tilde{m}_{\mathrm{r}}} \kappa_{n}(\omega) \bar{p}_{\ell n}^{(\infty)}\left(-L_{\mathrm{r}}, y, \omega\right)}{\sum_{n=1}^{\tilde{m}_{\mathrm{r}}} \bar{p}_{\ell n}^{(\infty)}\left(-L_{\mathrm{r}}, y, \omega\right)} \quad \text { for } \ell=0, j
$$

where the sum is truncated at a number $\widetilde{m}_{\mathrm{r}}$ less than the number of fluid modes $m_{\mathrm{r}}$ ensuring convergence.

Figs. 3 and 4 illustrate the results obtained for two reservoir truncation lengths $L_{\mathrm{r}}=0.1 H_{\mathrm{r}}$ and $L_{\mathrm{r}}=0.5 H_{\mathrm{r}}$, respectively. Error estimators are given in $\%$ and are 

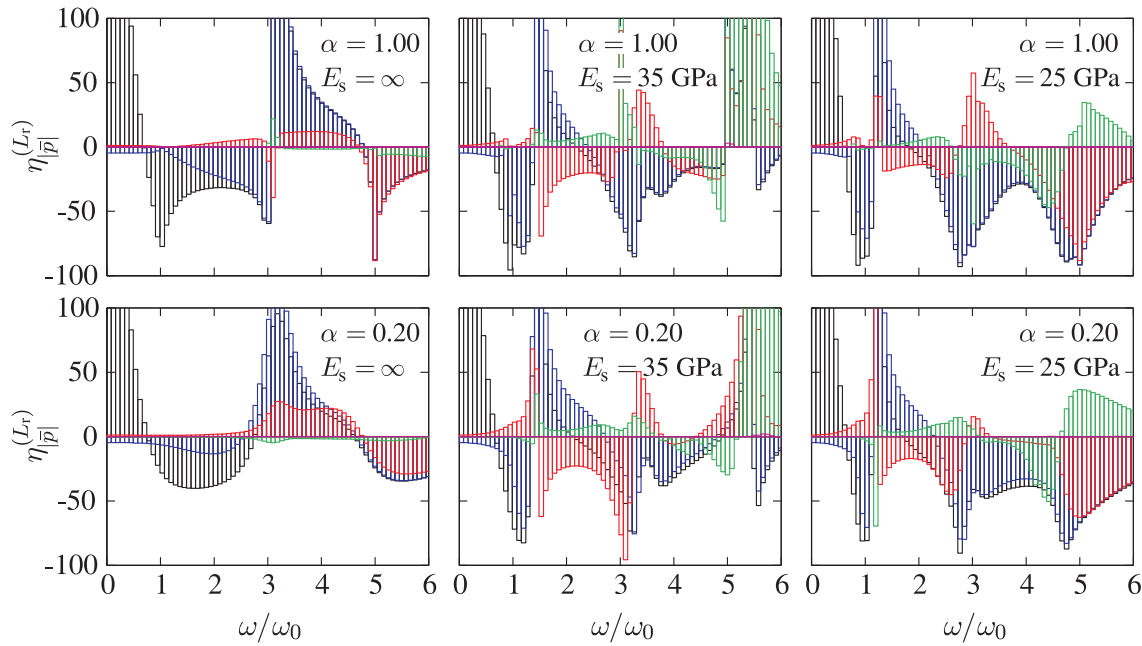

Figure 3: Hydrodynamic pressure error estimator for truncation length $L_{\mathrm{r}}=0.1 H_{\mathrm{r}}$. — Sommerfeld BC; __ Sharan BC; __ Analytical BC with $\widetilde{m}_{\mathrm{r}}=2$; Analytical BC with $\widetilde{m}_{\mathrm{r}}=3$; __ Analytical BC with $\widetilde{m}_{\mathrm{r}}=10$.

presented as bar charts equally spaced at frequency increments of $\Delta \omega=0.08 \omega_{0}$ over a frequency ratio range from 0 to 6 . For each truncation length, rigid and flexible dams with $E_{\mathrm{s}}=35 \mathrm{GPa}$ and $E_{\mathrm{s}}=25 \mathrm{GPa}$ are investigated, as well as two wave reflection coefficients $\alpha=1.0$ and $\alpha=0.2$, where $\alpha$ represents the portion of the wave amplitude reflected back to the reservoir and is related to the damping factor $q$ by [7]

$$
\alpha=\frac{1-q C_{\mathrm{r}}}{1+q C_{\mathrm{r}}}
$$

The bar charts in fig. 3 clearly indicate that the error associated with the Sommerfeld boundary condition is the highest for most of the frequency range of interest, namely for low frequencies $\omega \leqslant \omega_{0}$. It is seen that the Sharan boundary condition yields satisfactory results only for very low frequencies, i.e. a maximum error of approximately 5\%. The error associated with the Sharan boundary condition varies significantly as a function of frequency ratio and dam flexibility, but we note that this error globally increases with dam stiffness. Sharan and Sommerfeld boundary conditions yield similar error estimators in the higher frequency range. We also observe that error estimators are less sensitive to reservoir bottom wave absorbtion. More reservoir modes need to be included to match hydrodynamic pressures in the higher frequency range. The number of reservoir modes required for convergence in a rigid dam case, i.e. $\widetilde{m}_{\mathrm{r}} \approx 3$, is less than that required in the case of a flexible dam, i.e. $\widetilde{m}_{\mathrm{r}} \approx 10$. It is important to note however that an increase in the number of reservoir modes does not necessarily reduce the error estimators over the whole frequency range, but rather enlarges the lower frequency range over which error is minimum. No definite trend could be 

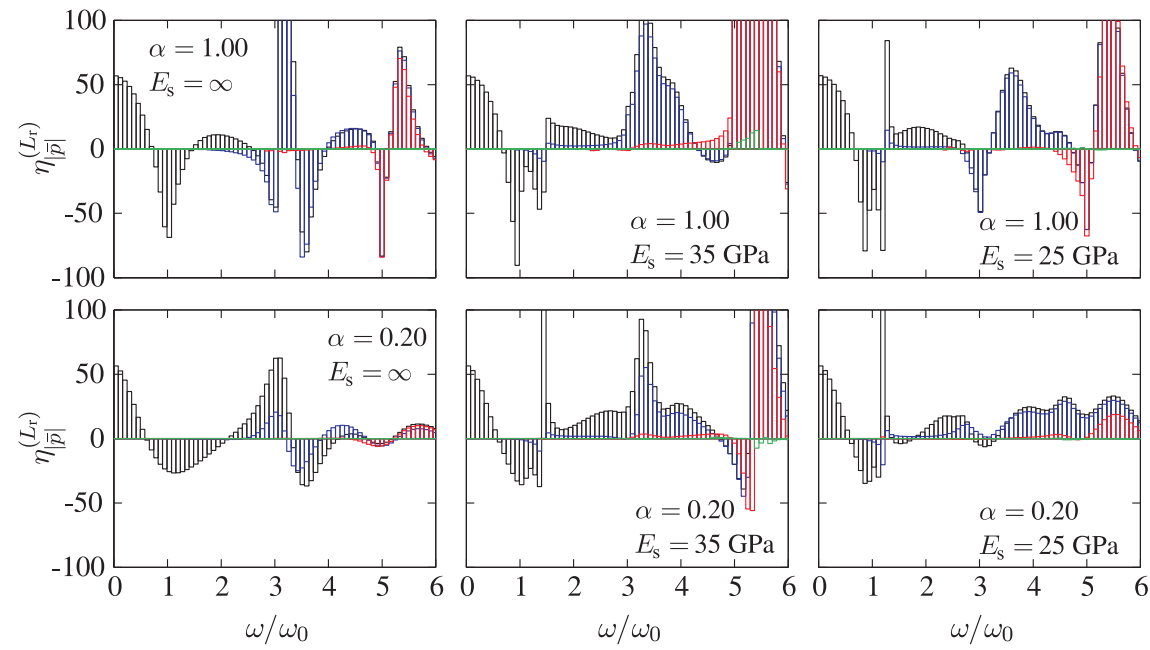

Figure 4: Hydrodynamic pressure error estimator for truncation length $L_{\mathrm{r}}=0.5 H_{\mathrm{r}}$. — Sommerfeld BC; __ Sharan BC; __ Analytical BC with $\widetilde{m}_{\mathrm{r}}=2$; Analytical BC with $\widetilde{m}_{\mathrm{r}}=3$; _ Analytical BC with $\widetilde{m}_{\mathrm{r}}=10$.

identified regarding the sign of the error estimators. For example, Sharan boundary condition is shown to be alternatively conservative or nonconservative depending on the frequency ratio.

As truncation length increases, fig. 4 shows that error estimators generally diminish over the whole frequency range. For low frequencies, Sommerfeld boundary condition still yields unsatisfactory results, while the error due to Sharan boundary condition is nearly null. Fewer reservoir modes are now required to obtain convergence over all the frequency range, i.e. $\widetilde{m}_{\mathrm{r}} \approx 3$. The effect of energy dissipation at reservoir bottom is slightly more important that for truncation length $L_{\mathrm{r}}=0.1 H_{\mathrm{r}}$. It is seen that reservoir bottom wave absorption causes error estimators to slightly diminish. As previously, Sharan and Sommerfeld yield approximately similar error estimators in the higher frequency range. We also observe that the error reduction due to reservoir bottom wave absorption becomes more pronounced with increasing truncation length. For the sake of brevity, the previous results focused on the evaluation of hydrodynamic pressure in a given point at dam-reservoir interface. Other error estimators for response quantities such as hydrodynamic forces or vibration periods can be obtained using the proposed formulation as detailed elsewhere [9].

\section{Concluding remarks}

This paper presented and validated an original formulation to study dynamically excited fluid-structure systems including TBCs. The mathematical derivations 
were provided and the resulting systems of equations were solved numerically to assess the accuracy and effectiveness of some classical approximate TBCs to determine the error associated with their use independently of FEM or BEM modeling of the reservoir. In an illustrative example of a dam-reservoir system, a proposed error estimator was investigated to get more insight into the sensitivity of hydrodynamic pressures at dam upstream face when a given TBC is applied at reservoir upstream. It is shown that the proposed formulation can be used efficiently for a rigorous assessment of the accuracy and effectiveness of classical or newly-developed TBCs, namely by defining reservoir length to depth ratios and frequency ranges for which the application of these TBCs is recommended. The proposed procedure, which can be easily programmed, is valuable in testing, validating or developing either frequency-dependent TBCs or frequency-independent TBCs generally more suited for time domain analyses.

\section{Acknowledgements}

The authors would like to acknowledge the financial support of the Natural Sciences and Engineering Research Council of Canada (NSERC).

\section{References}

[1] Westergaard, H. M., Water pressures on dams during earthquakes. Transactions ASCE, 98, 418-472, 1933.

[2] Saini, S. S., Bettess, P. \& Zienkiewicz, O. C., Coupled hydrodynamic response of concrete gravity dams using finite and infinite elements. Earthquake Engineering and Structural Dynamics, 6, 363-374, 1978.

[3] Sharan, S. K., Efficient finite element analysis of hydrodynamic pressure on dams, Journal of Computers and Structures, 42, 713-723, 1992.

[4] Bouaanani, N. \& Lu, F. Y., Assessment of potential-based fluid finite elements for seismic analysis of dam-reservoir systems, Journal of Computers and Structures, 87, 206-224, 2009.

[5] Çetin, M. \& Mengi, Y., Transmitting boundary conditions suitable for analysis of dam-reservoir interaction and wave load problems, Applied Mathematical Modelling, 27, 451-470, 2003.

[6] Gogoi, I. \& Maity, D., A non-reflecting boundary condition for the finite element modeling of infinite reservoir with layered sediment, Advances in Water Resources, 29, 1515-1527, 2006.

[7] Fenves, G. \& Chopra, A. K., Earthquake analysis and response of concrete gravity dams. Report No. EERC-84/10, Univ. of California, Berkeley, 1984.

[8] Sommerfeld, A., Partial differential equations in physics. Academic Press, New York, 1949.

[9] Bouaanani, N. \& Miquel, B., A new formulation and error analysis for vibrating dam-reservoir systems with upstream transmitting boundary conditions. Journal of Sound and Vibration, 329, 1924-1953, 2010. 